\title{
POLARIZATION OF RADIATION BY THE AEROSOL-GAS COMPONENT OF THE ATMOSPHERE FOR LIDAR WAVELENGTHS.
}

\author{
ANNA ZIMOVAYA ${ }^{1}$, ALEXANDER KONOSHONKIN²." \\ ${ }^{1}$ Laboratory of Optical Signals Propagation, V.E. Zuev Institute of Atmospheric Optics SB RAS, Tomsk, \\ 634055, Russia \\ ${ }^{2}$ Laboratory of Atmospheric Radiation, V.E. Zuev Institute of Atmospheric Optics SB RAS, Tomsk, 634055, \\ Russia
}

\section{*Email:avk@iao.ru}

\begin{abstract}
The report presents an original algorithm for solving the radiation transfer equation based on the Monte Carlo method for problems of atmospheric laser sensing, taking into account the polarization of radiation. A number of test calculations were performed to analyze the data of the polarization scanning lidar of the V.E. Zuev Institute of Atmospheric Optics SB RAS. It is shown that the proposed algorithm, taking into account the polarization, makes it possible to reduce the discrepancy in interpreting lidar data in comparison with the algorithm without taking into account polarization.
\end{abstract}

\section{INTRODUCTION}

In problems of atmospheric laser sensing, the interaction of radiation with aerosol is paid close attention. As a rule, to take into account the influence of the aerosol component, it is necessary to solve the radiation transfer equation. The most suitable method for solving the radiation transfer equation is the Monte Carlo method, since it allows us to perform calculations with controlled accuracy. For problems of polarization laser sensing, it is necessary to take into account the influence of polarization. The paper presents the original algorithm developed by the authors, which allows to solve the radiation transfer equation taking into account the polarization.

\section{METHODOLOGY}

The simulation of energy flow with allowance for radiation polarization in the considered formulation of the problem was performed by the Monte Carlo method with local estimates at points of collision by the backward scheme. In contrast to the kernel of the scalar integral radiation transfer equation (RTE), the kernel of vector integral RTE for the Stokes vector parameters is not self-conjugated, which introduces some specificity in comparison with the scalar case. For simulation of the radiation transfer with allowance for polarization by the backward scheme with the use of local estimates at every point of collision, it is necessary to store the product of scattering matrices at the previous collisions. That is, at every new collision the following matrix should be formed:

$$
\begin{gathered}
M_{i}=M_{i-1} \cdot P\left(\boldsymbol{\omega}^{\prime}, \boldsymbol{\omega}, \mathbf{r}\right) \\
P\left(\boldsymbol{\omega}^{\prime}, \boldsymbol{\omega}, \mathbf{r}\right)=L\left(\pi-i_{2}\right) R(\mu, \mathbf{r}) L\left(-i_{1}\right) \\
R(\mathbf{r}, \mu)=\frac{\sigma_{s, a}(\mathbf{r})}{\sigma_{t, a}(\mathbf{r})+\sigma_{t, m}(\mathbf{r})} \times \\
\times \frac{3}{4}\left(\begin{array}{cccc}
\frac{\left(1+\mu^{2}\right)}{2} & -\frac{\left(1-\mu^{2}\right)}{2} & 0 & 0 \\
-\frac{\left(1-\mu^{2}\right)}{2} & \frac{\left(1+\mu^{2}\right)}{2} & 0 & 0 \\
0 & 0 & \mu & 0 \\
0 & 0 & \mu
\end{array}\right)+ \\
+\frac{\sigma_{s, m}(\mathbf{r})}{\sigma_{t, a}(\mathbf{r})+\sigma_{t, m}(\mathbf{r})} \cdot\left(\begin{array}{cccc}
r_{11} & r_{12} & 0 & 0 \\
r_{12} & r_{22} & 0 & 0 \\
0 & 0 & r_{33} & r_{34} \\
0 & 0 & -r_{34} & r_{44}
\end{array}\right),
\end{gathered}
$$

where $\boldsymbol{r}$ is the radius-vector of the scattering point; $L$ is the rotation matrix; $R$ is the total scattering matrix; $\mathrm{P}$ is the phase scattering matrix; $\mu$ is the cosine of the scattering angle, $r_{11}, r_{12}, r_{33}, r_{34}$ are elements of the aerosol scattering matrix on the assumption of homogeneity and sphericity of aerosol particles [1], $i_{1}$ and $i_{2}$ are the angles between the scattering plane and the planes passing through the vertical at the point of 
collision and the vectors of direction before and after scattering, respectively.

Then, in local estimates, the received radiation is determined as a product of the obtained matrix $\mathrm{M}_{\mathrm{i}}$ and initial values of the Stokes vector-parameter. The values of elements of the aerosol scattering matrix depend on the state of aerosol and should be determined for every particular situation.

\section{MODEL OF THE ATMOSPHERE WITH ALLOWANCE FOR THE RADIATION POLARIZATION}

For development of efficient models of the atmosphere, NASA organized the AERONET network of stations for ground-based observation of the state of the atmosphere [2]. The data of measurement of the aerosol state are available at [2]. These data include the aerosol optical depth (AOD) of the atmosphere, complex refractive index of aerosol particles, particle size distribution, and scattering phase functions. The scattering phase functions are given only for wavelengths of $0.34,0.38,0.44,0.5,0.675,0.87$, 1.02 , and $1.64 \mu \mathrm{m}$. However, for operation with actual lidar data, it is necessary to have the scattering phase functions for the lidar wavelengths. Toward this end, the elements of the aerosol scattering matrix were calculated from the data on the complex refractive index and the particle size distribution by the program based on [3]. An example of calculation, the measurement date is shown in Fig. 1.

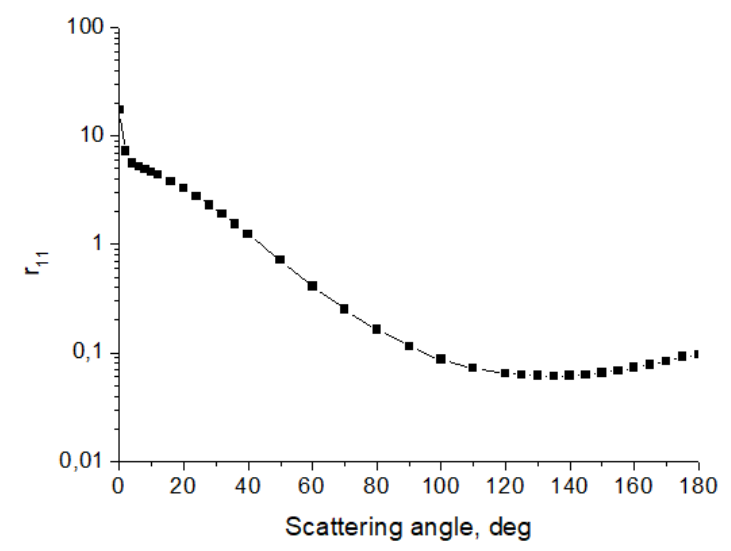

a)

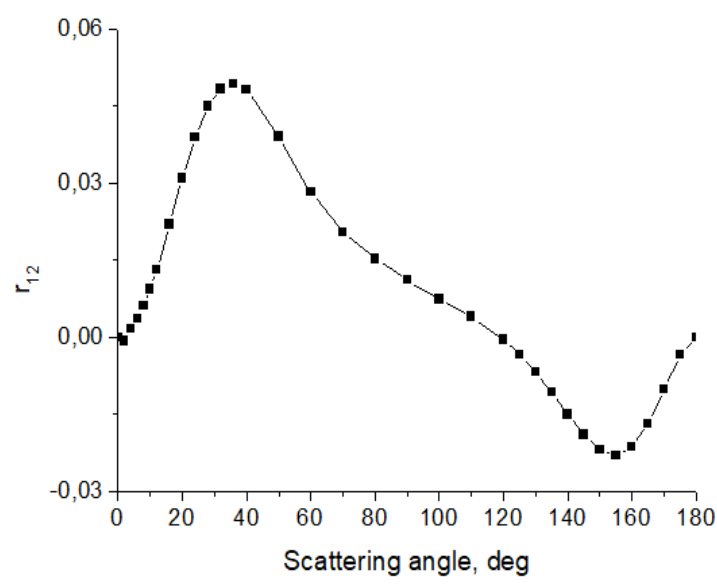

b)

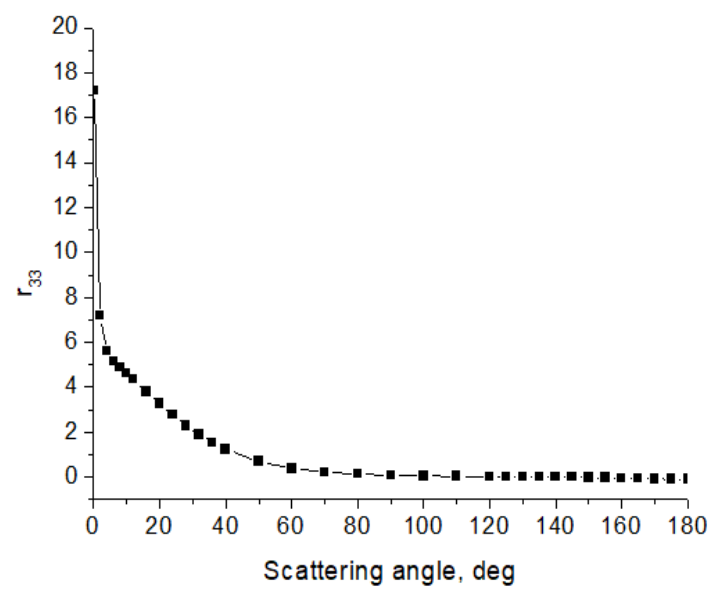

c)

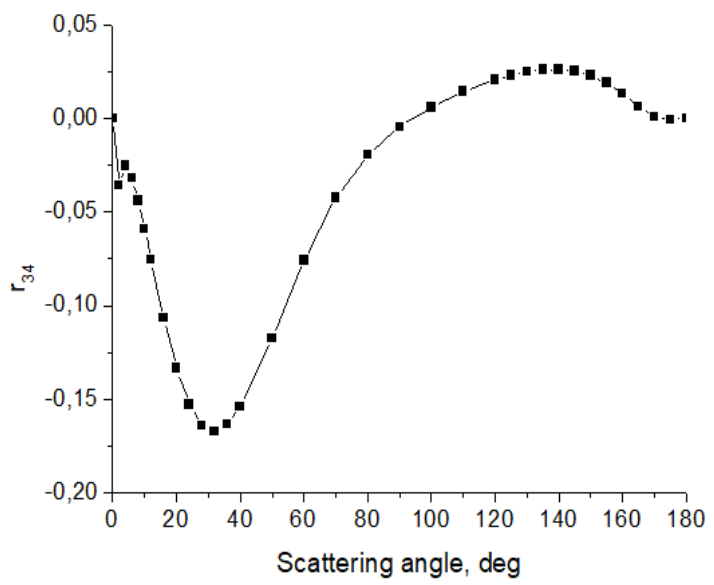

d) 


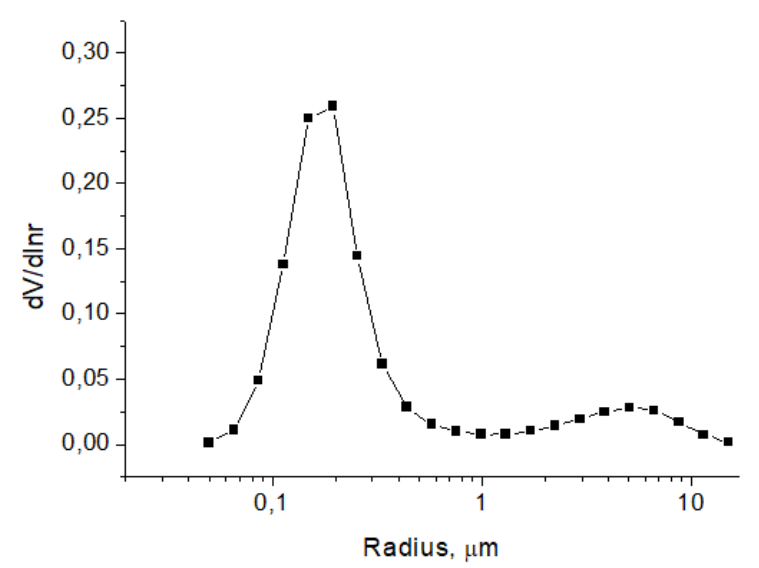

e)

Figure 1. a-d) elements of aerosol scattering matrix $r_{11}$, b) $r_{12}$, c) $r_{13}$, d) $r_{14}$, e) Particle size distribution function

\section{RESULTS OF NUMERICAL CALCULATIONS}

The proposed algorithm supplemented with the program for calculation of light scattered in atmosphere with polarization taken into account was tested for a southern area of the Tomsk Region with coordinates of $55.95-56.85^{\circ} \mathrm{N}$ and $84.05-84.95^{\circ} \mathrm{E}$ for scanning lidar of V.E. Zuev Institute of Atmospheric Optics. The correction was made for $\lambda=0.535 \mu \mathrm{m}, 1.064 \mu \mathrm{m}$. In the nearsurface layer (up to $5 \mathrm{~km}$ ), the aerosol scattering matrix was calculated from measurements of the AERONET station for the corresponding days. For the upper layers, the changing of polarization was neglected. The vertical profile of the optical coefficients of aerosol was set by the LOWTRAN-7 [4] model closest in AOD to the AERONET measurements. The point with the coordinate $56.5^{\circ} \mathrm{N}, 84.3^{\circ} \mathrm{E}$, was selected as the test point. The results of comparison of average errors of retrieval are shown in Figure 2.

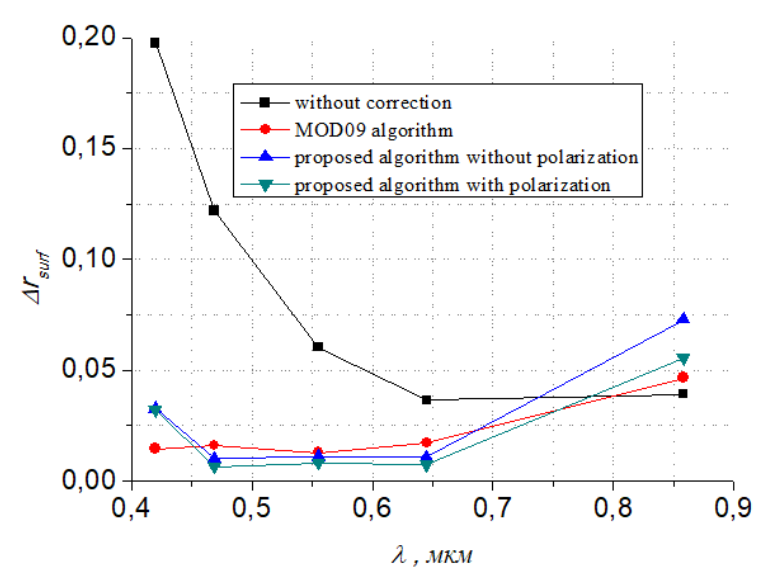

Figure 2. Comparison of average differences between algorithm without polarization and proposed algorithm with polarization.

The figure shows that the allowance for the radiation polarization and the use of AERONET measurements allow us to significantly decrease the error of the proposed algorithm. It can be important for lidar applications.

\section{REFERENCES}

[1] H. C. van de Hulst., Light scattering by small particles. By New York, London, 1957. Pp. 470;

[2] Aerosol Robotic Network (AERONET), http://aeronet.gsfc.nasa.gov.

[3] Bohren C.F., Huffman D.R., "Absorption and Scattering of Light by Small Particles," Wiley, 530 (1983).

[4] Kneizys F.X., Shettle E.P., Anderson G.P., Abreu L.W., Chetwynd J.H., Selby J.E.A., Clough S.A., Gallery W.O. User Guide to LOWTRAN-7. - ARGLTR-86-0177. ERP 1010. Hanscom AFB. 1988. MA $01731.137 \mathrm{p}$. 